\title{
Development and characterization of a novel nanoemulsion drug-delivery system for potential application in oral delivery of protein drugs
}

This article was published in the following Dove Press journal:

International Journal of Nanomedicine

25 October 2012

Number of times this article has been viewed

\author{
Hongwu Sun $1, *$ \\ Kaiyun Liu',* \\ Wei Liu' \\ Wenxiu Wang ${ }^{2}$ \\ Chunliang Guol \\ Bin Tang' \\ Jiang $\mathrm{Gu}^{\prime}$ \\ Jinyong Zhang' \\ Haibo $\mathrm{Li}^{\prime}$ \\ Xuhu Mao' \\ Quanming Zou' \\ Hao Zeng'
}

'National Engineering Research Center for Immunological Products, Third Military Medical University, Chongqing, China; ${ }^{2}$ Key Lab of Preventive Veterinary Science and Animal Biological Technology, Shandong Binzhou Animal Science and Veterinary Medicine Institute, Binzhou, China

*These authors contributed equally to this work.
Correspondence: Quanming Zou/Hao Zeng

National Engineering Research Center for Immunological Products and Department of Microbiology and Biochemical Pharmacy, College of Pharmacy, Third Military Medical University, 30 Sha Ping Ba Gaotanyan Street, Chongqing 400038, China

Tel +8623687523I5

Fax +862368753590

Email qmzou2007@।63.com/

zenghao2008@yahoo.com.cn
Background: The stability of protein drugs remains one of the key hurdles to their success in the market. The aim of the present study was to design a novel nanoemulsion drug-delivery system (NEDDS) that would encapsulate a standard-model protein drug - bovine serum albumin (BSA) - to improve drug stability.

Methods: The BSA NEDDS was prepared using a phase-inversion method and pseudoternary phase diagrams. The following characteristics were studied: morphology, size, zeta potential, drug loading, and encapsulation efficiency. We also investigated the stability of the BSA NEDDS, bioactivity of BSA encapsulated within the NEDDS, the integrity of the primary, secondary, and tertiary structures, and specificity.

Results: The BSA NEDDS consisted of Cremophor EL-35, propylene glycol, isopropyl myristate, and normal saline. The average particle diameter of the BSA NEDDS was about $21.8 \mathrm{~nm}$, and the system showed a high encapsulation efficiency $(>90 \%)$ and an adequate drugloading capacity $(45 \mathrm{mg} / \mathrm{mL})$. The thermodynamic stability of the system was investigated at different temperatures and $\mathrm{pH}$ levels and in room-temperature conditions for 180 days. BSA NEDDS showed good structural integrity and specificity for the primary, secondary, and tertiary structures, and good bioactivity of the loaded BSA.

Conclusions: BSA NEDDS showed the properties of a good nanoemulsion-delivery system. NEDDS can greatly enhance the stability of the protein drug BSA while maintaining high levels of drug bioactivity, good specificity, and integrity of the primary, secondary, and tertiary protein structures. These findings indicate that the nanoemulsion is a potential formulation for oral administration of protein drugs.

Keywords: nanoemulsion, drug-delivery system, protein drug, oral administration, stability

\section{Introduction}

Many protein targets and protein drugs have recently been discovered, with approximately a dozen therapeutic protein drugs receiving regulatory approval each year around the world. ${ }^{1,2}$ Over 125 protein drugs are already in the US market, and the value of advanced drug-delivery systems in this market had reached $\$ 121$ billion by $2010 .^{3}$ Although many varieties of protein drugs have been designed for therapeutic purposes, several challenges remain, including enzymatic susceptibility, stability during storage period, and efficacy after administration. ${ }^{4}$ Thus, protein stability is one of the major hurdles for a drug's successful entry into the market. ${ }^{5}$ At present, many of the marketed protein drugs are mainly designed for injective delivery. Because of the thermal instability of protein drugs, they need to be stored and transported at cold temperatures in order to ensure long shelf life. ${ }^{5,6}$ 
To overcome the crucial problem of protein instability, stabilizers such as nonionic surfactants, cyclodextrin derivatives, poloxamers, glycerol, ethylene glycol, glucose, sucrose, and trehalose have been used. ${ }^{7}$ However, the effects of these stabilizers vary among different proteins. With a view to enhancing the stability of therapeutic peptides and proteins, many studies have focused on the use of such drug-delivery systems as microspheres, nanoparticles, liposomal systems, and traditional emulsions. ${ }^{8}$ However, these drug-delivery systems have several drawbacks, such as low encapsulation efficiency and drug loading, use of inorganic solvents such as methylene chloride and acetone (which induce toxicity), low yield and high production costs. ${ }^{8}$ A nanoemulsion-drug delivery is a novel, thermodynamically stable delivery system that can enhance the stability of a protein drug by protecting it from oxidative and enzymatic degradation. Bovine serum albumin (BSA) has often been used as a standard and model protein drug in drug development and delivery, using carriers such as microparticles, nanospheres, nanoparticles, and gels. ${ }^{9-13}$

The nanoemulsion drug-delivery system (NEDDS) is an isotropic system in which two immiscible liquids are combined to form a single phase by application of appropriate surfactants or by mixing to form a droplet with a diameter range of approximately $0.1-100 \mathrm{~nm} .{ }^{14}$ Such systems can also be formed by heterogeneous dispersion of oil droplets in an aqueous medium stabilized using surfactant molecules. ${ }^{15}$ In recent years, the focus on NEDDS has increased because of its distinctive structure and properties, including a small droplet size, possibility of fabrication with biocompatible materials, increased dissolution rate and solubility, improved diffusion across an unstirred aqueous layer, and enhanced mucosal permeability. ${ }^{16}$ NEDDS possesses high kinetic and long-term thermodynamic stability and transparency. Therefore, it has been widely used to improve the stability of drugs such as ramipril, ${ }^{17}$ celecoxib, ${ }^{18}$ hyaluronic acid, ${ }^{19,20}$ $\beta$-cypermethrin, ${ }^{21} \beta$-carotene, ${ }^{22,23} \beta$-cyclodextrin ${ }^{24}$ and Bacillus anthracis protective antigen. ${ }^{25}$

NEDDS possesses a good storage capacity and thermodynamic stability for drug delivery. In this study, we chose BSA as our model drug. And also, we prepared and optimized BSA NEDDS by using a phase-inversion method and pseudoternary phase diagrams. The delivery system was evaluated using transmission electron microscopy, atomic force microscopy, Nano ZS90 (Malvern Instruments, Malvern, UK). We also tested the following characteristics of the system stored at room temperature for 180 days: thermodynamic stability, specificity, bioactivity and stability of this system and model protein drug, BSA, including integrity of the primary, secondary, and tertiary structures.

\section{Materials and methods Materials}

BSA (MW 66 kDa; fat, purity 98.3\%; lot 071K7602) was purchased from Sigma-Aldrich Chemicals (St Louis, MO) and used without further purification. Isopropyl myristate (IPM) was purchased from Croda (Goole, UK). Cremophor EL 35 (polyethoxylated castor oil) was obtained from BASF (Mumbai, India). Propylene glycol and Tween 20 were obtained from Shanghai Chemical Reagent Corporation (Shanghai, China). Low molecular protein ladder marker (14.4-97.4 kDa) and predye protein ladder marker $(17-170 \mathrm{kDa})$ were purchased from Fermentas International (Burlington, Canada). A micro-BCA protein assay kit was purchased from Wuhan Boster Bioengineering, Wuhan, China. Rabbit anti-mouse BSA monoclonal antibody was purchased from Beijing Saichi Shengwu Keji (Beijing, China). Horseradish peroxidase (HRP) goat anti-mouse immunoglobulin $\mathrm{G}$ ( $\mathrm{IgG}$ ) was purchased from Beijing Zhongshan Golden Bridge Biotechnology (Beijing, China). High-purity Milli-Q water (Millipore, Billerica, MA) was used for all solutions, with a resistance value of $>18.2 \mathrm{MV} / \mathrm{cm}$. All other chemicals used in the study were of analytical reagent grade.

\section{Pseudoternary phase diagram construction}

On the basis of the results of preliminary experiments, we chose IPM as the oil phase, Cremophor EL-35 as the surfactant, propylene glycol as the cosurfactant, and normal saline as the aqueous phase. The surfactant and the cosurfactant were mixed (Smix) in different mass ratios $(1: 1,2: 1$, and $3: 1)$. The pseudoternary phase diagrams for these mixtures were created using titration at room temperature to ascertain the ideal concentration ranges of the components. Nine different combinations of oil and Smix $(1: 9,2: 8,3: 7,4: 6,5: 5,6: 4,7: 3,8: 2$, and 9:1) were added drop-wise to the aqueous phase by using gentle agitation. Transparent and easily flowing nanoemulsion was observed. Using Origin 7.0 software (OriginLab, Northampton, MA), we generated the physical state of the nanoemulsion by creating a pseudo-three-component phase diagram, with the first axis representing the aqueous phase, the second axis representing the oil, and the third axis representing the mixture of the surfactant and cosurfactant at a fixed mass ratio. 


\section{Formulation of the BSA NEDDS}

From the pseudoternary diagram and visual observations, a precisely defined composition was selected for the nanoemulsion-drug delivery system. Briefly, Smix was prepared by mixing Cremophor EL 35, propylene glycol in the ratio $2: 1(\mathrm{w} / \mathrm{w})$ and the mixture was stirred at $600 \mathrm{rpm}$ at $25^{\circ} \mathrm{C}$. Next, IPM was added to Smix to obtain the desired ratio of 9:1 (Smix-IPM) and stirred for 5 minutes, after which the mixture was cooled to room temperature. This was followed by the addition of aqueous phase (normal saline) to the Smix-IPM mixture while stirring, such that the final concentration of aqueous phase in the mixture was $20 \% \mathrm{w} / \mathrm{w}$. The contents were stirred for 15 minutes to ensure nanoemulsion formation, which was monitored by checking the optical clarity and physical properties of the system. To prepare the novel BSA NEDDS, a blank formula and different concentrations of BSA power and aqueous solution, being 5, 10, $15,20,25,30,35,40,45,50,55$, and $60 \mathrm{mg} / \mathrm{mL}$, were used following the same protocol and method to the pseudoternary phase diagram construction, with the exception of water being replaced by BSA as the aqueous phase.

\section{Characterization of the BSA NEDDS}

Sample solutions were imaged using transmission electron microscopy (Tecnai-10 TEM; Hillsboro, OR). A drop of the particle suspension was placed onto a carbon-coated copper grid obtained from Ted Pella (Redding, CA) to yield a thin liquid film. The films were negatively stained with $1 \%$ phosphotungstic acid solution (w/w; $\mathrm{pH}$ 7.4) for 1 minute. A high-resolution atomic-force microscope (IPC-208B; Chongqing University, Chongqing, China) was used to characterize the molecular morphology of the BSA NEDDS. Approximately $0.05 \mathrm{~mL}$ of the sample solution was dropped onto microscopic glass slides with gold coating to fracture the film, which was then dried and tested. The measurement was performed under the following conditions: tungsten probes (force constant, $0.06 \mathrm{~N} \cdot \mathrm{m}$ ); scan range, $10.5 \mathrm{~nm} \times 10.5 \mathrm{~nm}$; imaging mode, tapping mode; and scanning method, point by point scanning at room temperature. The G3DR software (Changqing, China) was used to process the data. The excess phosphotungstic solution was removed using filter paper, and stained samples were characterized at $80 \mathrm{kV}$. The average size and zeta potential of the BSA NEDDS were tested at $20^{\circ} \mathrm{C}$ by using a dynamic light-scattering instrument (Zetasizer) equipped with an argon ion laser.

\section{Drug-loading and encapsulation efficiency}

For sample preparation, triplicate aliquots of the blank and novel BSA NEDDS (1 g, approximately, $1 \mathrm{~mL}$ ) were placed in sterile 2-mL eppendorf centrifuge tubes and diluted to a protein concentration of $250-500 \mathrm{mg} / \mathrm{mL}$ with normal saline. Two volumes of absolute ethanol were added to approximately $0.3 \mathrm{~mL}$ of blank or sample, and the mixture was shaken to ensure complete emulsification, then centrifuged at $13,000 \times g$ for 30 minutes. The supernatant was removed, and the precipitate was mixed with $0.3 \mathrm{~mL}$ of the above solutions. The amount of BSA incorporated in the nanoemulsion was determined by centrifuging the BSA-loaded nanoemulsion and separating the supernatant. The precipitate was discarded, and the BSA content of the solution was then analyzed by using the commercially available bicinchoninic acid kit. The following equations were used in calculating for the percentage of drug loading and encapsulation efficiency:

Drug loading $(\%)=($ Total weight of protein drug/Total weight of sample) $\times 100 \%$

Encapsulation efficiency $(\%)=($ Actual $/$ Theoretical protein drug loading) $\times 100 \%$

\section{Stability study of the BSA NEDDS Thermodynamic stability}

Thermodynamic stability was tested and revised according to the methods described by Shafiq et al. ${ }^{26}$ Briefly, selected formulations were centrifuged at $13,000 \times g$ for 30 minutes at $4^{\circ} \mathrm{C}$ and $25^{\circ} \mathrm{C}$, maintain for 48 hours. Six cycles of centrifugation were performed, and the appearance of these formulations was investigated by using indexes including turbidity, phase separation, precipitation, drug separation, demulsification, and creaming.

\section{Effects of different temperatures and $\mathrm{pH}$ values}

Effects of the temperatures and $\mathrm{pH}$ on particle size and zeta potential of the BSA NEDDS were measured directly. Effects of the increasing temperature in the range $20^{\circ} \mathrm{C}-80^{\circ} \mathrm{C}$ on particle size were determined by Nano ZS90 at $20^{\circ} \mathrm{C}$. Effects of increasing $\mathrm{pH}$ in the range 1-14 on particle size and zeta potential were measured by Nano ZS90 at $20^{\circ} \mathrm{C}$.

\section{Short- and long-term stability}

To examine the physical stability of this system, short- and long-term stability studies were carried out on the selected formulations. Samples were divided into three vials after production and stored at room temperature for 180 days. On days $0,30,60,90$, and 180, all samples were examined and centrifuged for 30 minutes at $13,000 \times g$. Turbidity, phase separation, precipitation, drug separation, breaking, and creaming were evaluated, and also the primary structural 
integrity was again assessed by sodium dodecyl sulfate polyacrylamide gel electrophoresis. Furthermore, the main physical characteristics, including particle size, polydispersity index, zeta potential, electrophoresis mobility, and conductivity were determined using photon correlation spectroscopy on days $0,30,60,90$, and 180 at $20^{\circ} \mathrm{C}$.

\section{Stability study of the BSA enclosed in the BSA NEDDS}

\section{Relative content and bioactivity assay}

The relative content and bioactivity of four samples stored at room temperature for $0,30,90$, and 180 days were determined. BSA was extracted from the nanoemulsion by using the drug-loading and encapsulation efficiency protocol described previously. The BSA content of the BSA NEDDS, which were stored at room temperature for $0,30,90$, and 180 days, were detected using microbicinchoninic acid methods. Relative bioactivity was determined by enzymelinked immunosorbent assay. The methods were performed as follows: 96-well flat-bottom plates (Costar, WA) were coated overnight at $4{ }^{\circ} \mathrm{C}$ with $50 \mu \mathrm{g} / \mathrm{mL}$ of theoretical protein drug above four samples, blocked with a blocking buffer $(10 \mathrm{mM}$ PBS, pH 7.4, with $0.05 \%$ Tween 20), and kept at overnight at $4{ }^{\circ} \mathrm{C}$. Goat anti-mouse BSA monoclonal antibody (1:300) was used as the primary antibody. And HRP-conjugated goat anti-mouse $\operatorname{IgG}(1: 10,000)$ was used as the secondary antibody. $O$-phenylenediamine was used as a substrate. The absorbance of the solutions was then determined using BioRad model 680 microplate reader (Bio-Rad, Hercules, CA) set at a wavelength of $490 \mathrm{~nm}$.

\section{Evaluation of primary structural integrity and specificity}

To determine the primary structural integrity and specificity of the BSA NEDDS, sodium dodecyl sulfate polyacrylamide gel electrophoresis and Western blotting were performed. The supernatant and precipitate of a blank nanoemulsion, the supernatant and precipitate of the BSA nanoemulsion, the supernatant and precipitate of a BSA emulsion, and native BSA aqueous solution were separated using 10\% sodium dodecyl sulfate polyacrylamide gel electrophoresis. The gels were electroblotted onto polyvinylidene fluoride filter membranes at $25 \mathrm{mV}$ for 15 minutes by using a TransBlot semidry blotter (Bio-Multiphor II electrophoresis unit; GE Healthcare, Amersham, UK), after which they were blocked using a buffer (10 mM PBS, pH 7.4 with $0.05 \%$ Tween 20 ) and kept overnight at $4^{\circ} \mathrm{C}$. Subsequently, the membranes were hybridized with goat anti-mouse BSA monoclonal antibody $(1: 300)$ for 1 hour at $37^{\circ} \mathrm{C}$. HRP-conjugated goat anti-mouse IgG was used as the secondary antibody (1:10,000). Binding reaction was detected using diaminobenzidine color-development reagent $(0.05 \%$ diaminobenzidine and $0.005 \%$ hydrogen peroxide in distilled water).

\section{Evaluation of the secondary structure}

To verify the integrity of the secondary structure of the BSA in this NEDDS, circular dichroic spectra of the BSA aqueous solution and three batches of the BSA NEDDS placed at room temperature for 30,90 , and 180 days were acquired at room temperature by using a Chirascan spectropolarimeter (Jasco 810, Easton, MD) equipped with ultraviolet light (190-250 nm) with a $0.01^{-\mathrm{cm}}$ path length and a step size of $1 \mathrm{~nm}$. The lamp housing was purged with nitrogen, and an average of three scans was obtained. BSA was extracted from the NEDDS by using the previously mentioned protocol.

\section{Evaluation of the tertiary structure}

To verify the integrity of the tertiary structure of BSA in this NEDDS, we used Fourier transform infrared (FTIR) spectroscopy. FTIR spectra of the BSA aqueous solution and three batches of the BSA NEDDS stored at room temperature for 30, 90, and 180 days were obtained using a Spectrum One spectrophotometer (Lambda 950; PerkinElmer, Waltham, MA) separately 500, 550, 600, and 200 times diluting with water. Four samples were prepared using the potassium bromide disk method and examined using the transmission mode. A spectral range of $400-4000 \mathrm{~cm}^{-1}$ with a resolution of $2 \mathrm{~cm}^{-1}$ (accumulation of 64 scans) was used in order to obtain good-quality spectra. By monitoring the peak frequencies and locations of certain bands, all possible chemical changes and interactions were determined.

\section{Statistical analysis}

Values are expressed as means \pm standard deviation. Statistical significance was determined using one-way analysis of variance by the least-significant-difference test. $P<0.05$ was considered significantly different.

\section{Results}

\section{Construction of pseudoternary phase diagrams}

Pseudoternary phase diagrams were separately constructed for each Smix ratio (Figure 1). A Smix with a ratio $(\mathrm{Km})$ of 2:1 in a mixture of Cremophor EL-35 (surfactant), propylene glycol (cosurfactant), isopropyl myristate (oil), and normal saline (aqueous medium) showed a large nanoemulsion area 


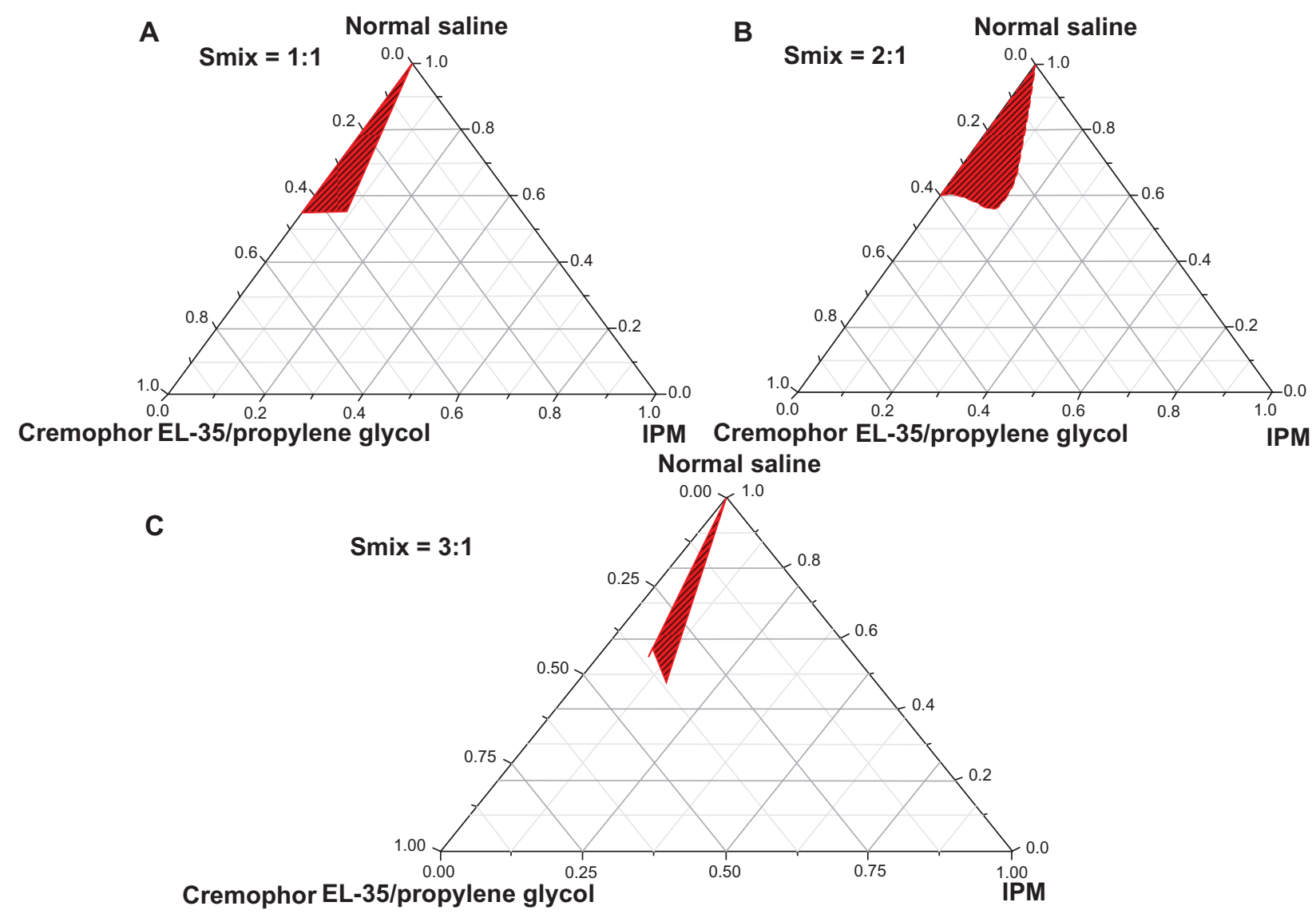

Figure I Pseudoternary phase diagrams of the BSA NEDDS, composed of the following constituents: oil, isopropyl myristate; surfactant, Cremophor EL-35; and cosurfactant, propylene glycol; drug-loading value was $45 \mathrm{mg} / \mathrm{mL}$. (A) Surfactant and cosurfactant mixture ratio (Smix), I:I; (B) Smix, 2:1; (C) Smix, 3:1.

Note: The red area represents the nanoemulsion delivery system region.

Abbreviations: BSA, bovine serum albumin; NEDDS, nanoemulsion drug-delivery system; IPM, isopropyl myristate.

(Figure 1B). Figure 1C shows that the 3:1 Smix formula generated the smallest nanoemulsion area. The area of nanoemulsion increased with Smix proportions of $1: 1$ and 2:1 (Figure 1A and B). On the basis of these pseudoternary phase diagrams, we chose the Smix of Cremophor EL-35/ propylene glycol at a ratio of $2: 1$, which showed the largest nanoemulsion area, as the main formulation for the BSA NEDDS.

\section{Preparation of the BSA NEDDS formulation}

It is important to determine the surfactant concentration accurately and to use the optimum concentration in the formulation. According to the results of the different concentrations and dosages of BSA, the BSA aqueous solution was added to the mixture of Cremophor EL-35, propylene glycol, and isopropyl myristate. BSA (45 mg/mL) was dissolved in the optimized mixture of $18 \%$ Cremophor EL-35, $9 \%$ propylene glycol, $3 \%$ isopropyl myristate (all w/w) and slowly added to normal saline under gentle stress to produce a transparent nanoemulsion-delivery system using the phaseinversion method.

\section{Characterization of the BSA NEDDS}

The droplets in the novel BSA NEDDS appeared dark and the surroundings were bright, such that a "positive" image was observed using transmission electron microscopy (Figure 2A). We randomly selected 500 droplets on the electron micrograph, and calculated the average particle size according to the scale and droplet-diameter measurements and statistical data. The results showed that the droplet particle sizes were mostly within the range of $1-100 \mathrm{~nm}$, with an average size of approximately $21.0 \mathrm{~nm}$. Examination of the atomic-force microscopy images revealed that most of the droplets exhibited spherical morphology, with a mean diameter of approximately $20 \mathrm{~nm}$ (Figure 2B). The droplets were solid, with surfaces mostly rugged with sags and crests. No droplets aggregated, especially in the BSA stabilized with the nanoemulsion, suggesting its stability, although proteins in the neighboring droplets might have been in contact with each 

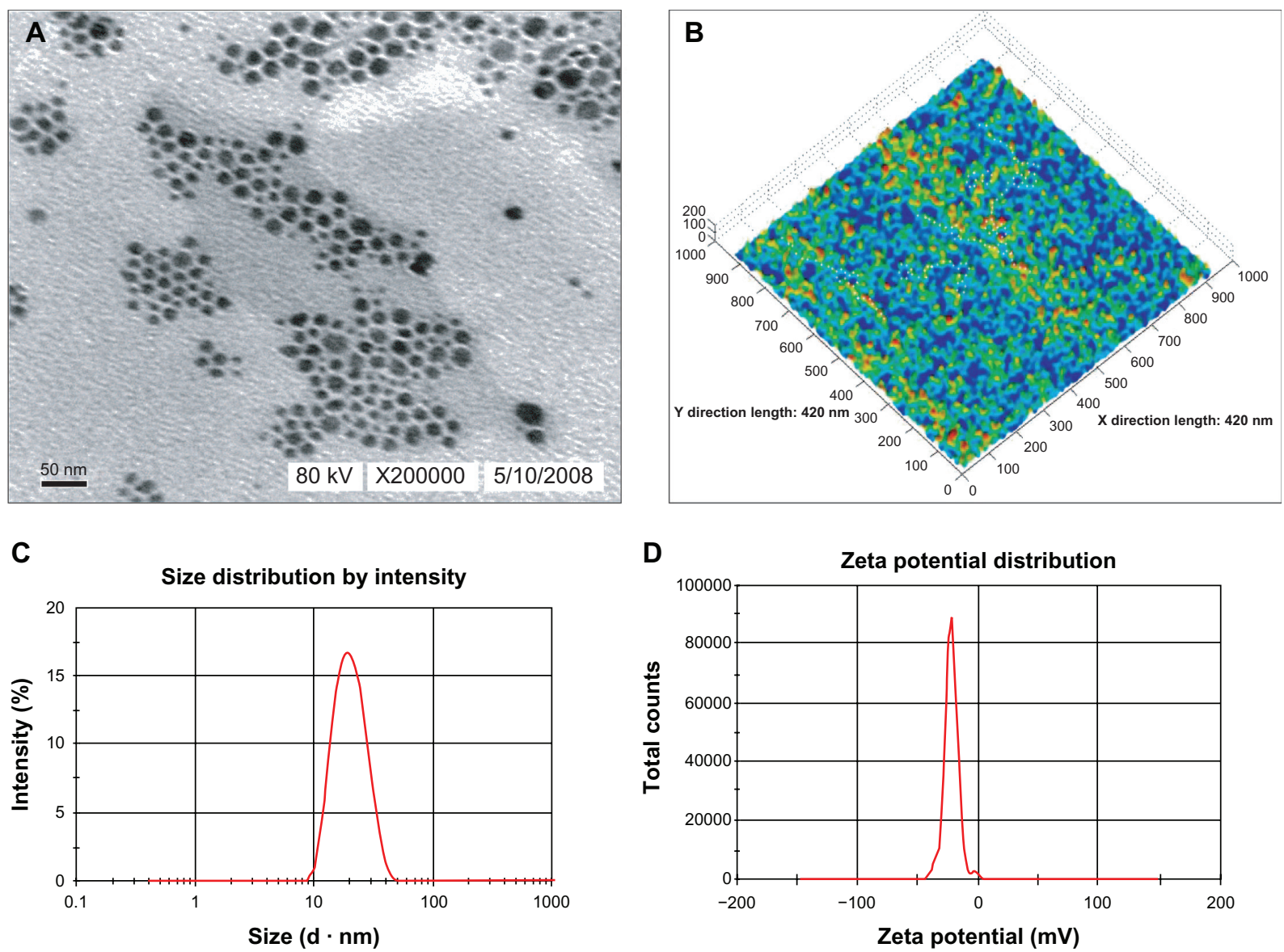

Figure 2 (A-D) Characteristics of the BSA NEDDS. (A) Transmission electron micrograph of the BSA NEDDS; (B) atomic force microscopy; (C) particle-size distribution; (D) zeta-potential distribution.

Abbreviations: BSA, bovine serum albumin; NEDDS, nanoemulsion drug-delivery system.

other. The differences observed among these morphological evaluations were essentially due to the fact in the Nano ZS90, the hydrodynamic radius of the particle was measured. On the contrary, transmission electron microscopy and atomic-force microscopy were performed on nanoemulsions of surface ions and solvent and nonsolvent molecules. Consequently, the actual mean diameter (obtained by transmission electron microscopy) was generally far smaller than the hydrodynamic diameter. Figure $2 \mathrm{C}$ shows that the average particle size in the novel BSA NEDDS was $21.8 \mathrm{~nm}, 75 \%$ of the particles were $<50 \mathrm{~nm}$ and $90 \%<60 \mathrm{~nm}$ in size, and the range of distribution was very narrow. We determined the polydispersity index (PDI) value, which measures the spread of the particle-size distribution, in which a small value indicates a narrow particle-size range. ${ }^{22}$ In general, the novel BSA NEDDS exhibited a relatively limited size distribution, with a PDI value of 0.170 , indicating a close size distribution and good stability of the nanoemulsion due to the reduced Ostwald ripening. Figure 2D shows that the average particle size in the novel BSA NEDDS was $-24.21 \mathrm{mV}$ and remained in the $-40-\mathrm{mV}$ to $40-\mathrm{mV}$ range. The viscosity, refractive index, and dispersant refractive index were $0.8872 \mathrm{cP}, 1.59$ $\mathrm{n}_{\mathrm{D}}{ }^{20}$, and 1.330 , respectively.

\section{Drug-loading and encapsulation efficiency}

The bicinchoninic acid method is a basic, classical technique used to detect BSA content. The standard curve equation is $y=0.0008 x+0.1636, r^{2}=0.9948$, and the linear range is $25-1000 \mu \mathrm{g} / \mathrm{mL}$. The drug-loading content and encapsulation efficiency of the BSA NEDDS can be measured in comparison to a standard curve. In this study, we obtained high encapsulation efficiency $(>90 \%)$ and enough drug loading $(45 \mathrm{mg} / \mathrm{mL})$.

\section{Stability study of the BSA NEDDS}

\section{Thermodynamic stability}

After six cycles, the BSA NEDDS did not show any phase separation, turbidity, precipitation, drug separation, demulsification, or creaming. These observations suggest that this system has good thermodynamic stability at temperatures 
between $4^{\circ} \mathrm{C}$ and $25^{\circ} \mathrm{C}$. Thermostability also differentiates nanoemulsion from emulsions, which are kinetically stable and eventually show phase separation. Therefore, the formulations obtained in our study were tested for thermodynamic stability by using centrifugation and heating-cooling and freeze-thaw cycles. Thermodynamic stability analysis showed no turbidity, phase separation, precipitation, drug separation, demulsification, or creaming before or after centrifugation $(13,000 \times g, 30$ minutes $)$ within the temperature range of $4^{\circ} \mathrm{C}-25^{\circ} \mathrm{C}$.

\section{Effects of different temperatures and $\mathrm{pH}$ values}

Effects of the increasing temperatures or $\mathrm{pH}$ values on the particle size and zeta potential were determined using the Nano ZS90, as shown in Figure 3. Figure 3A shows the effect of increasing temperature on particle size of the BSA NEDDS. The particle size did not change significantly (it remained in the $20-30-\mathrm{nm}$ range) at temperatures up to $80^{\circ} \mathrm{C}$ and increased rapidly at higher temperatures $\left(>80^{\circ} \mathrm{C}\right)$, but remained under $100 \mathrm{~nm}$. Figure 3B shows that no obvious change in zeta potential was observed within the $\mathrm{pH}$ range of $1-13$, and the value remained between $-40 \mathrm{mV}$ and $20 \mathrm{mV}$. The average particle size of the novel BSA NEDDS remained at $20-30 \mathrm{~nm}$ at different $\mathrm{pH}$ values (Figure $3 \mathrm{~B})$. These results showed that the average particle size did not obviously change on using high temperature up to $80^{\circ} \mathrm{C}$ (Figure $3 \mathrm{~A}$ ) and that the zeta potential remained in the $-40-\mathrm{mV}$ to $40-\mathrm{mV}$ range at different $\mathrm{pH}$ values (Figure $3 \mathrm{~B}$ ). The average particle size of the novel BSA NEDDS remained within the range of $20-30 \mathrm{~nm}$ at different $\mathrm{pH}$ values (Figure 3B).

\section{Short- and long-term stability}

The stability test showed that the novel BSA NEDDS did not change in visible appearance (ie, flocculation, stratification, precipitation, or creaming and emulsification) either
A

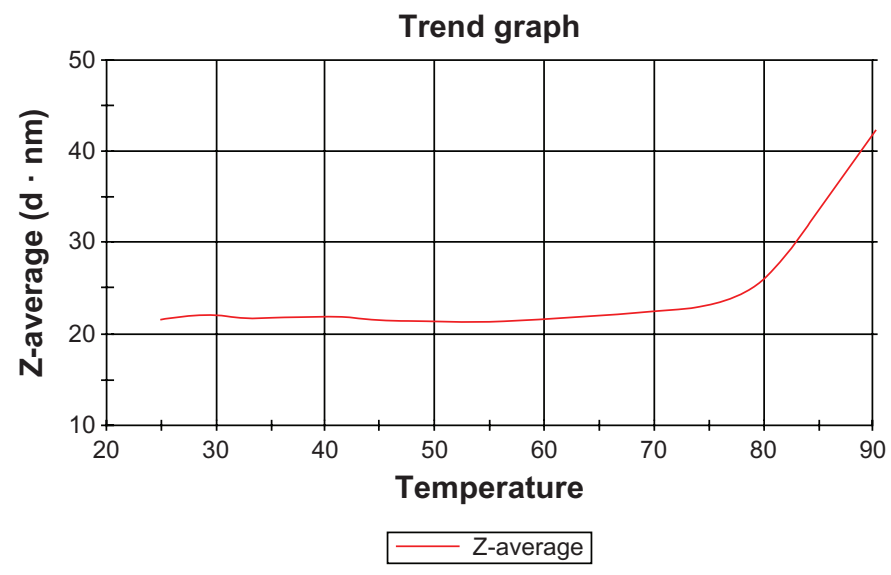

\begin{tabular}{|c|c|}
\hline $\begin{array}{c}\text { Temp } \\
{ }^{\circ} \mathrm{C}\end{array}$ & $\begin{array}{c}\text { Z-avg } \\
\text { d.nm }\end{array}$ \\
\hline 25.0 & 21.81 \\
28.0 & 22.16 \\
31.0 & 22.09 \\
34.0 & 21.89 \\
37.0 & 21.82 \\
40.0 & 22.00 \\
43.0 & 21.78 \\
46.0 & 21.54 \\
49.0 & 21.49 \\
52.0 & 21.34 \\
55.0 & 21.44 \\
58.0 & 21.46 \\
61.0 & 21.79 \\
64.0 & 22.03 \\
65.0 & 22.22 \\
70.0 & 22.57 \\
73.0 & 22.91 \\
76.0 & 23.71 \\
79.0 & 25.02 \\
82.0 & 28.74 \\
85.0 & 33.85 \\
88.0 & 38.91 \\
90.0 & 42.35 \\
\hline
\end{tabular}

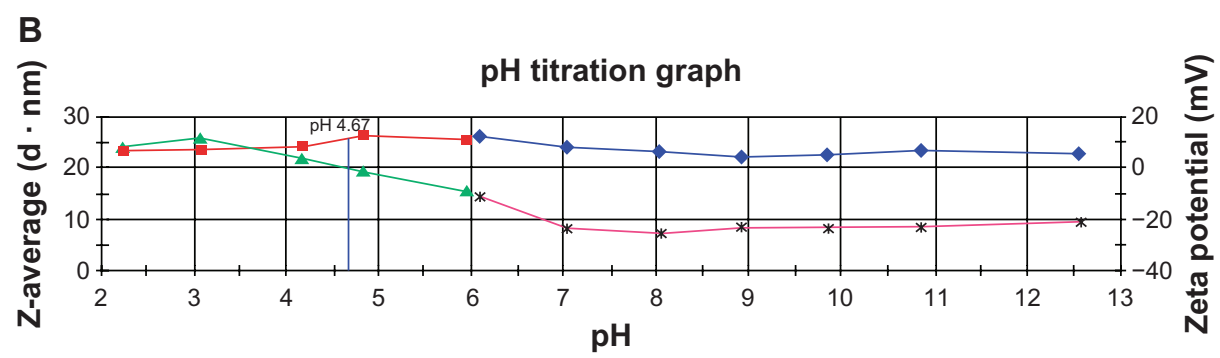

\begin{tabular}{|ll|}
\hline & Z-average (BSA nanoemulsion delivery system-less than $\mathrm{pH} 6.0$ titration) \\
- & Weighted mean Z-average (BSA nanoemulsion delivery system-less than $\mathrm{pH} 6.0$ titration) \\
- & Isoelectric point (BSA nanoemulsion delivery system-less than $\mathrm{pH} 6.0$ titration) \\
- & Z-average (BSA nanoemulsion delivery system-more than $\mathrm{pH} 6.0$ titration) \\
- & Weighted mean Z-average (BSA nanoemulsion delivery system-less than $\mathrm{pH} 6.0$ titration) \\
- & Z-potential (BSA nanoemulsion delivery system-less than $\mathrm{pH} 6.0$ titration) \\
- & Weighted Zeta pontential (BSA nanoemulsion delivery system-less than $\mathrm{pH} 6.0$ titration) \\
$*$ & Zeta potential (BSA nanoemulsion delivery system-more than $\mathrm{pH} 6.0$ titration) \\
- & Weighted mean Z-average (BSA nanoemulsion delivery system-more than $\mathrm{pH} 6.0$ titration) \\
\hline
\end{tabular}

Figure 3 (A and B) Effect of the different conditions on the BSA NEDDS. (A) Effect of particle size on the increasing temperature of the BSA NEDDS; (B) Effect particle size and zeta potential on the increasing $\mathrm{pH}$ values of the BSA NEDDS.

Abbreviations: BSA, bovine serum albumin; NEDDS, nanoemulsion drug-delivery system. 
in the natural state or after high-speed centrifugation $(13,000 \times g, 30$ minutes $)$. The main physical characteristics results - particle size, polydispersity index, condition, zeta potential, electrophoresis mobility and melting point - measured using the Nano ZS90 at $20^{\circ} \mathrm{C}$ are shown in Figure 4A-F. The BSA NEDDS, which was stored at room temperature for $0,30,60,90$, and 180 days, was very stable, and the particle size of this system was within the range of 20-30 nm (Figure 4A). Fluctuations in particle size were very small, and there was no change observed even after storage at room temperature for 180 days. The zeta potential remained between $-20 \mathrm{mV}$ and $-30 \mathrm{mV}$ (Figure 4B). Melting point and mobility and condition (Figure 4C-E) results showed no obvious changes either. There were also no visible changes in the main physical characteristics of the BSA NEDDS.

Figure 5A shows that the sodium dodecyl sulfate polyacrylamide gel electrophoresis gels of the BSA aqueous solution and this system stored at room temperature for 30 , 60, 90, and 180 days generated a clear band in lanes 1-5. At the same time points, BSA aqueous solution showed no obvious bands in lanes 6-9. These results imply that no changes occurred in the BSA NEDDS when it was stored at room temperature for 180 days.

\section{Stability study of the BSA in the BSA NEDDS}

\section{Relative content and bioactivity analysis}

Figure 5B showed no differences in the relative content among the fresh sample and the samples stored at room temperature for 30,60, 90, and 180 days. The relative content and bioactivity of these samples did not show any decrease after storage at room temperature for 180 days. The results showed that BSA in the BSA NEDDS has very high stability. BSA retained high bioactivity when the BSA NEDDS was stored at room temperature for 180 days.

Primary structural integrity and structural specificity The results of the evaluation of structural integrity are shown in Figure 6A, which shows that the aqueous BSA solution (lane 10), the supernatant nanoemulsion (lane 6), and the BSA emulsion (lane 8) each had one bright, clear band, each with a molecular weight of $66.4 \mathrm{kDa}$. The supernatant and precipitate of the blank nanoemulsion or emulsion
A
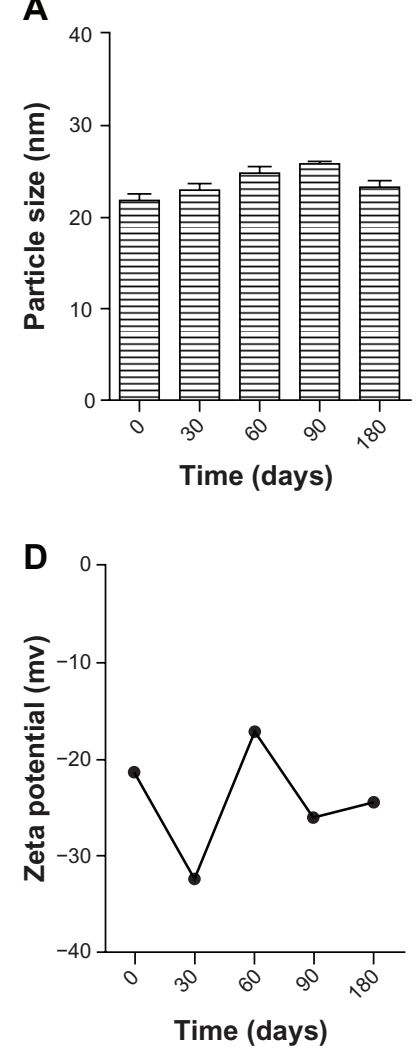

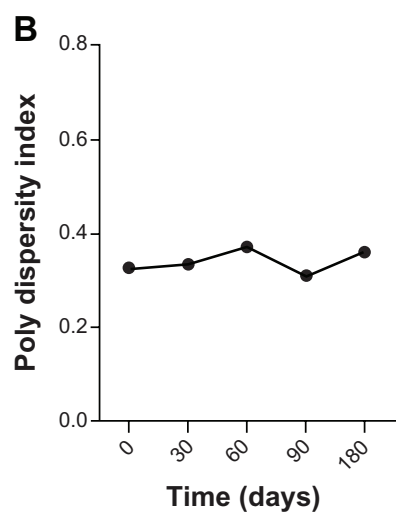

E

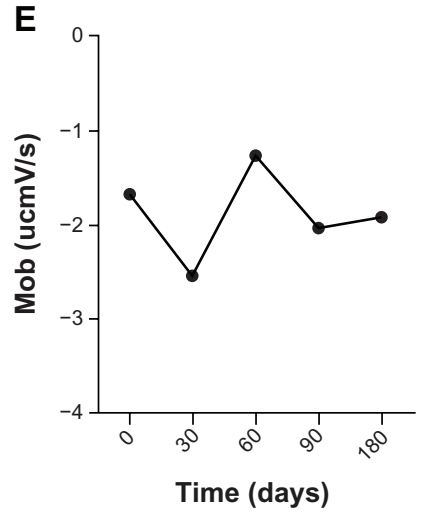

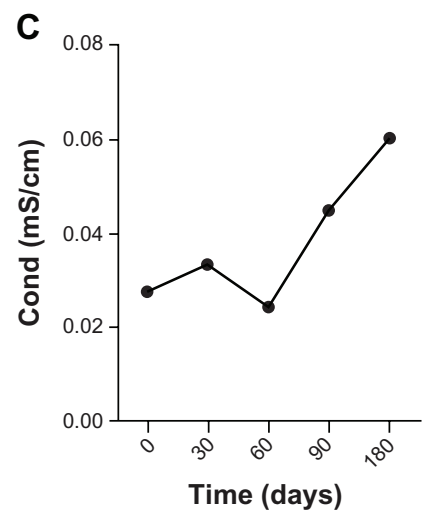

$\mathbf{F}$

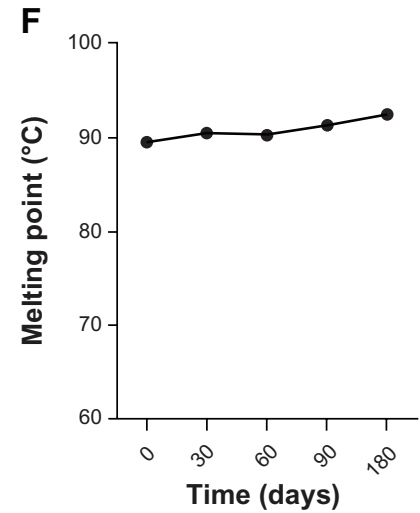

Figure 4 (A-F) Long-term stability results for the BSA NEDDS. (A) Particle size; (B) polydispersity index; (C) conductivity; (D) zeta potential; (E) electrophoresis mobility; (F) melting point.

Abbreviations: BSA, bovine serum albumin; NEDDS, nanoemulsion drug-delivery system. 


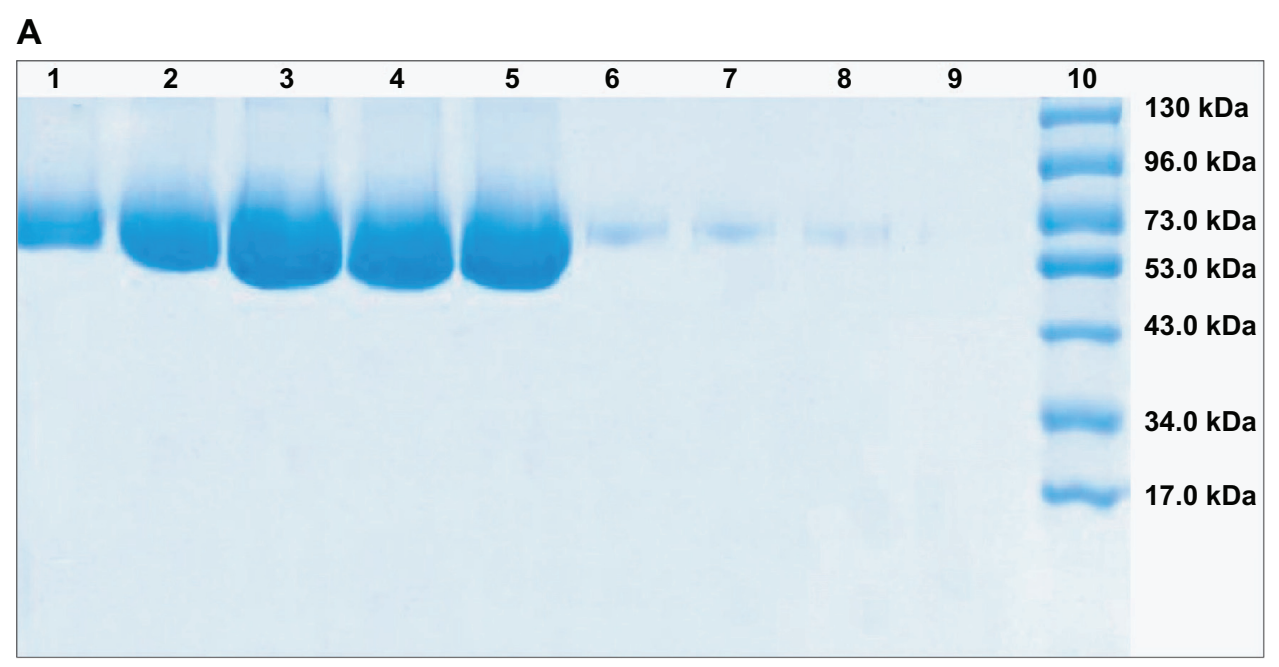

B

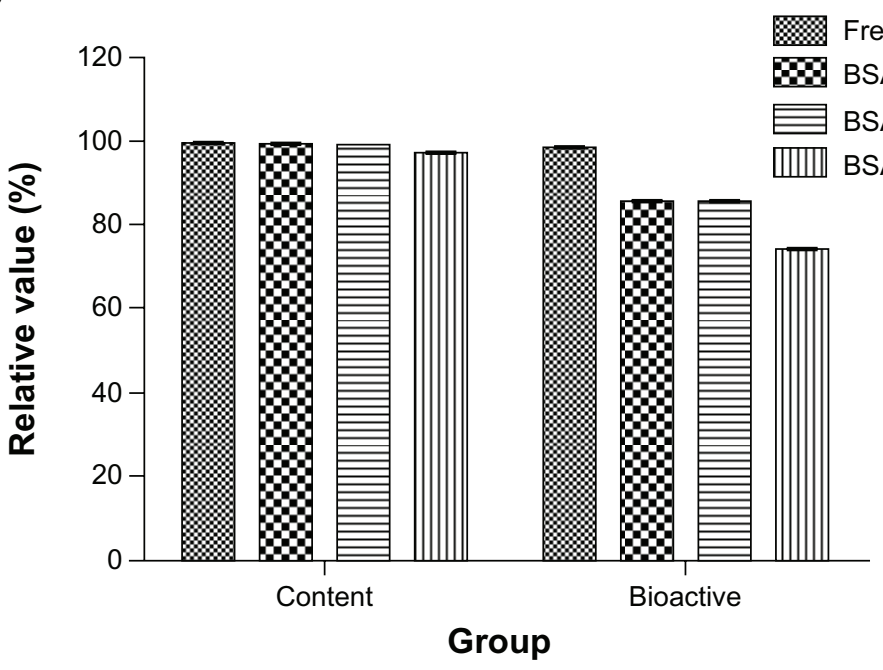

Figure 5 (A and B) Long-term stability results for BSA in the NEDDS. (A) Primary structural integrity changes in BSA in the NEDDS when stored at room temperature for 180 days. Lane I, BSA aqueous solution; lane 2, BSA NEDDS stored at room temperature for 30 days; lane 3, BSA NEDDS stored at room temperature for 60 days; lane 4, BSA NEDDS stored at room temperature for 90 days; lane 5, BSA NEDDS stored at room temperature for 180 days; lane 6, BSA aqueous solution stored at room temperature for 30 days; lane 7, BSA aqueous solution stored at room temperature for 60 days; lane 8 , BSA aqueous solution placed at room temperature for 90 days; lane 9 , BSA aqueous solution stored at room temperature for 180 days; lane 10, prestained protein ladder. (B) Relative content and bioactivity of the BSA in the nanoemulsion delivery system stored at room temperature for 180 days.

Note: No obvious changes in relative content and bioactivity were observed between the fresh samples and BSA NEDDS stored at room temperature for 30,90 , and I80 days. Abbreviations: BSA, bovine serum albumin; NEDDS, nanoemulsion drug-delivery system.

had no obvious bands (lanes 1-4). The precipitate of this nanoemulsion-drug delivery system (lane 7) and the emulsion (lane 9) of BSA had one very narrow and dark band at $66.4 \mathrm{kDa}$. The results for lanes 7 and 9 show that the surfactant and cosurfactant partly precipitate the protein, but the supernatants of the nanoemulsion and emulsion gave more intense bands, so most of BSA in the nanoemulsion and emulsion was present in the supernatant. Meanwhile, the results showing that the supernatant and precipitate of the blank nanoemulsion or emulsion had no band showed that sodium dodecyl sulfate polyacrylamide gel electrophoresis can be adapted to the evaluation of structural integrity.
Thus, the structural integrity of the novel BSA NEDDS was well maintained and showed no obvious degradation. In the structural specificity evaluation by Western blotting (Figure 6B), the protein lanes are similar to those observed in Figure 6A, and these bands are close to the 66-kDa markers. Once again, one clear band was evident in the BSA aqueous solution (lane 10), the nanoemulsion supernatant (lane 6), and the BSA emulsion (lane 8), while the supernatant and precipitate from the blank nanoemulsion and emulsion had no obvious bands (lanes 1-4). The precipitates of the novel BSA NEDDS (lane 7) and emulsion (lane 9) lacked clear bands. Meanwhile, the results showing that the supernatant 
and precipitate of the blank nanoemulsion or emulsion (lanes 1-4) had no bands suggested that Western blotting methods can also be adapted to the evaluation of structural specificity. Overall, good structural integrity and specificity of the BSA NEDDS are demonstrated in Figure 6A and B. For example, no obvious degradation was detected in the BSA NEDDS, but the supernatant and precipitate of the blank nanoemulsion and emulsion lacked obvious bands (Figure 6A and B). The supernatant of the nanoemulsion and emulsion showed obvious, intact bands (Figure 6A and B). These results showed good immunogenicity of the novel BSA NEDDS without obvious degradation of BSA.

\section{Integrity of the secondary structure}

To correlate the small changes in the aromatic environment observed with a possible change in the secondary structure of BSA, we assessed circular dichroic spectra in the ultraviolet region, which corresponds to the protein backbone. Figure 7A-D shows the circular dichroic spectrum of the BSA aqueous solution in comparison with the circular dichroic spectrum of the BSA extracted from nanoemulsion stored at room temperature for 30, 90, and 180 days. Both spectra showed clear minima of a similar magnitude at $209 \mathrm{~nm}$ and $222 \mathrm{~nm}$, which are indicative of the known $\alpha$-helical conformation of BSA. The reduced maximum at $210 \mathrm{~nm}$ indicated a relative loss of the helical structure of BSA following encapsulation and extraction from this NEDDS. In addition, the ratio of $\alpha$-helix, $\beta$-sheets, turns and random is not changed compared with that of the native aqueous BSA solution in Figure 8, in the secondary structure. These results suggest that BSA underwent adsorption at the interface within the emulsion system, which is commonly associated with some degree of unfolding. Importantly, the similarity characterize peak was about $210 \mathrm{~nm}$ and also its main secondary structure ratio of $\alpha$-helix and $\beta$-sheets did not change between the native aqueous BSA solution and its NEDDS when stored at room temperature for 180 days in the circular spectra. Turn and random of BSA showed part change compared the BSA NEDDS with native aqueous BSA solution.

\section{Integrity of the tertiary structure}

The BSA stability of the tertiary structure was measured by FTIR spectroscopy. In Figure 9 and Table 1, FTIR spectra of BSA aqueous solutions showed peaks at $3445.54 \mathrm{~cm}^{-1}, 2073.25 \mathrm{~cm}^{-1}, 1651.09 \mathrm{~cm}^{-1}, 1543.11 \mathrm{~cm}^{-1}$, $1455.79 \mathrm{~cm}^{-1}, 1403.38 \mathrm{~cm}^{-1}, 1308.93 \mathrm{~cm}^{-1}, 1245.23 \mathrm{~cm}^{-1}$,

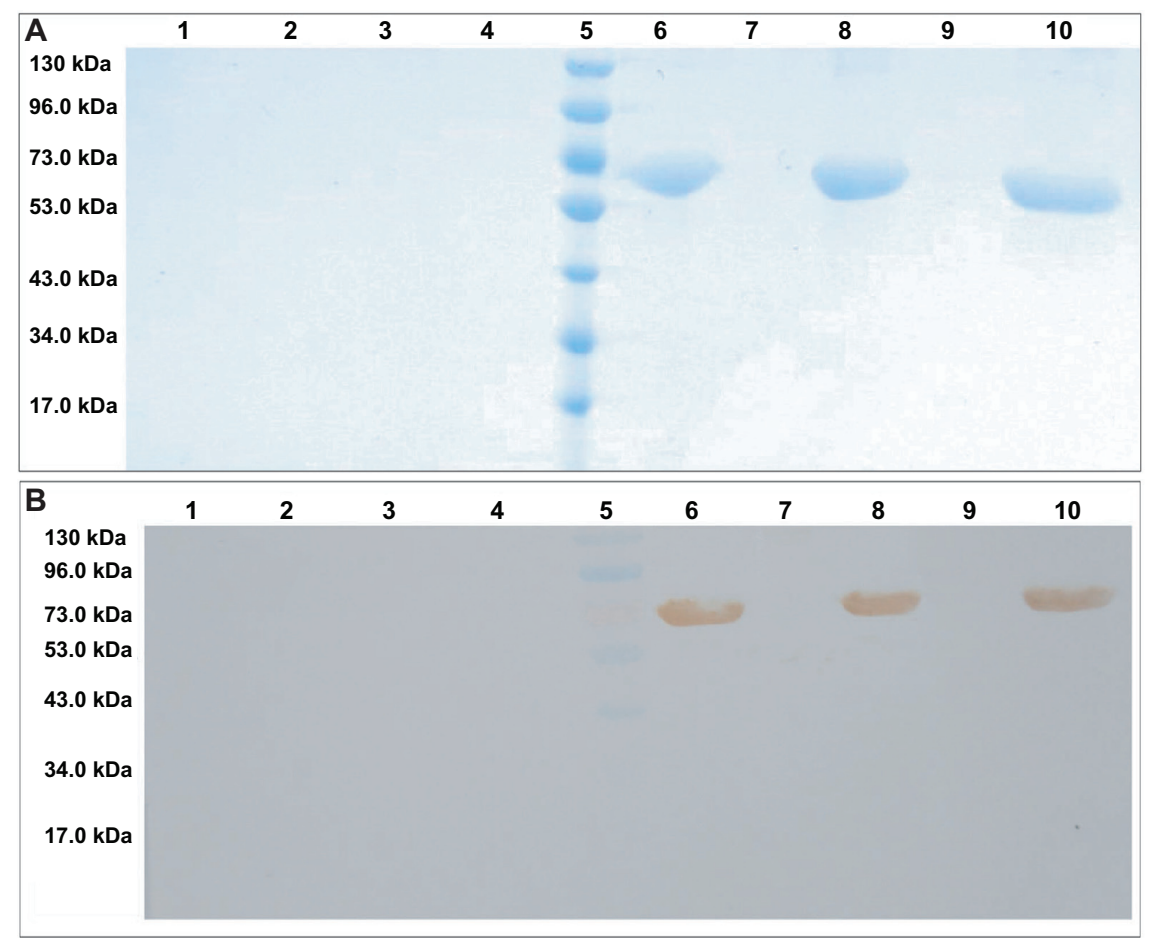

Figure 6 (A and B) Primary structural integrity and specificity results for the BSA in the NEDDS. (A) Sodium dodecyl sulfate polyacrylamide gel electrophoresis of BSA; (B) Western bolt analysis of BSA. Lane I, supernatant of blank nanoemulsion; lane 2, precipitate of blank nanoemulsion; lane 3, supernatant of blank emulsion; lane 4, precipitate of blank emulsion; lane 5, prestained protein ladder; lane 6, supernatant of the BSA nanoemulsion; lane 7, precipitate BSA nanoemulsion; lane 8 , supernatant of BSA emulsion; lane 9, precipitate of BSA emulsion; lane 10, BSA aqueous solution.

Abbreviations: BSA, bovine serum albumin; NEDDS, nanoemulsion drug-delivery system. 

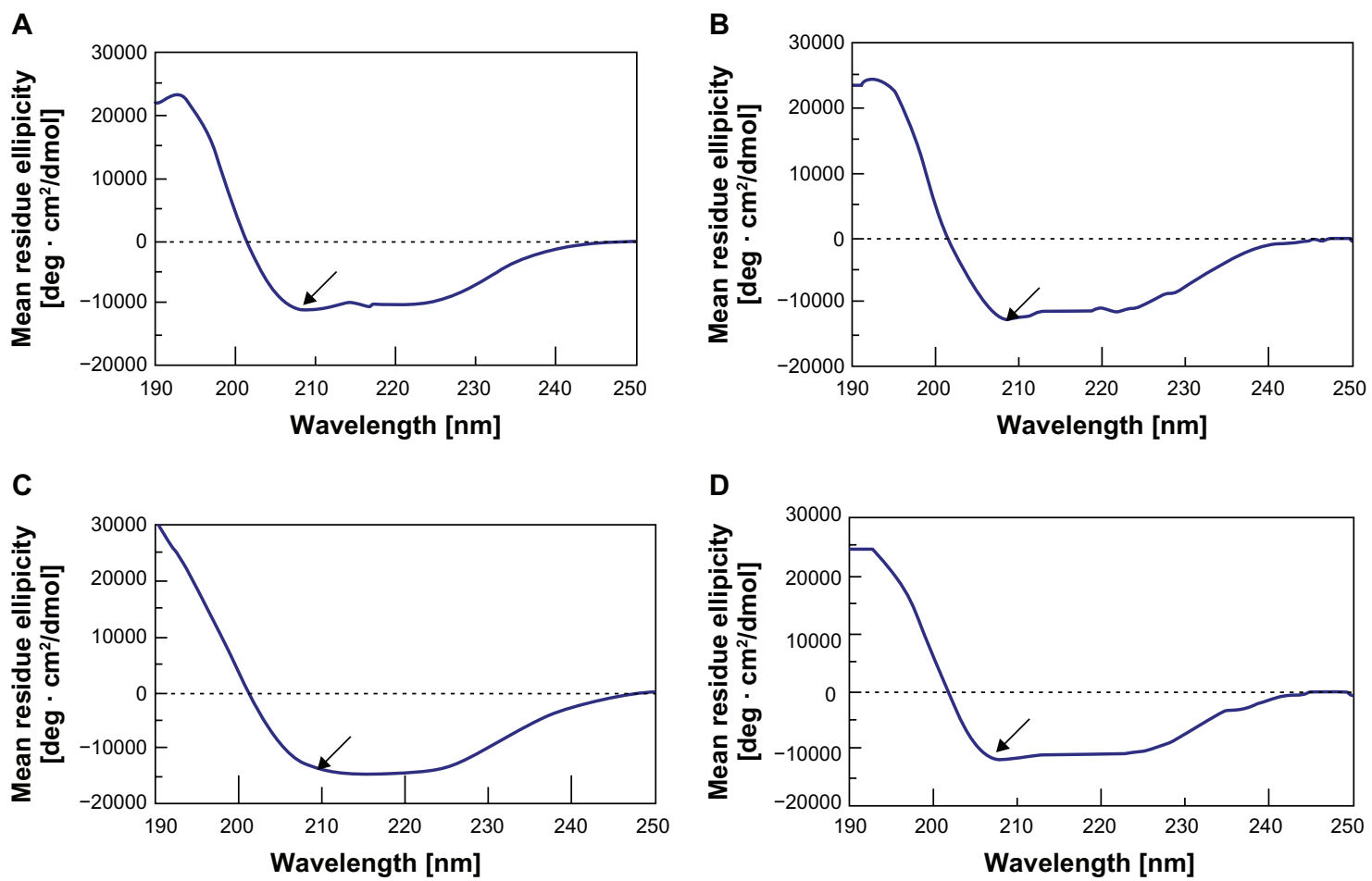

Figure 7 (A-D) Secondary structural spectra of the BSA in the NEDDS. (A) BSA aqueous solution; (B) BSA NEDDS stored at room temperature for 30 days; (C) BSA NEDDS stored at room temperature for 90 days; (D) BSA NEDDS stored at room temperature for 180 days.

Note: Arrows represent BSA absorption peaks.

Abbreviations: BSA, bovine serum albumin; NEDDS, nanoemulsion drug-delivery system.

$1162.07 \mathrm{~cm}^{-1}, 1114.44 \mathrm{~cm}^{-1}, 1019.18 \mathrm{~cm}^{-1}, 409.45 \mathrm{~cm}^{-1}$, and $401.15 \mathrm{~cm}^{-1}$. The BSA NEDDS stored at room temperature for 30 days showed peaks at $3445.98 \mathrm{~cm}^{-1}$, $2960.90 \mathrm{~cm}^{-1}, 2928.27 \mathrm{~cm}^{-1}, 1643.72 \mathrm{~cm}^{-1}, 1547.08 \mathrm{~cm}^{-1}$, $1513.11 \mathrm{~cm}^{-1}, 1455.28 \mathrm{~cm}^{-1}, 1388.11 \mathrm{~cm}^{-1}, 1351.77 \mathrm{~cm}^{-1}$,

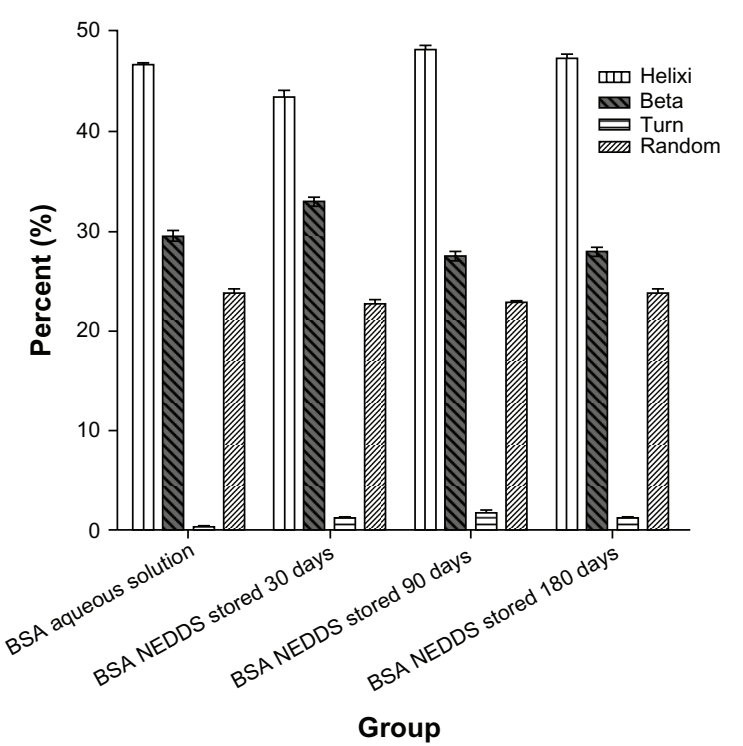

Figure 8 Secondary structural integrity results for the BSA in the NEDDS. Abbreviations: BSA, bovine serum albumin; NEDDS, nanoemulsion drug-delivery system.
$1293.05 \mathrm{~cm}^{-1}, 1249.53 \mathrm{~cm}^{-1}, 1181.92 \mathrm{~cm}^{-1}, 1103.03 \mathrm{~cm}^{-1}$, $943.77 \mathrm{~cm}^{-1}$, and $445.17 \mathrm{~cm}^{-1}$. The BSA NEDDS stored at room temperature for 90 days showed peaks at $3423.61 \mathrm{~cm}^{-1}, 2975.64 \mathrm{~cm}^{-1}, 2924.37 \mathrm{~cm}^{-1}, 2888.64 \mathrm{~cm}^{-1}$, $1924,15 \mathrm{~cm}^{-1}, 1651.09 \mathrm{~cm}^{-1}, 1512.45 \mathrm{~cm}^{-1}, 1454.77 \mathrm{~cm}^{-1}$, $1420.07 \mathrm{~cm}^{-1}, 1383.24 \mathrm{~cm}^{-1}, 1332.75 \mathrm{~cm}^{-1}, 1269.24 \mathrm{~cm}^{-1}$, $1185.99 \mathrm{~cm}^{-1}, 1088.44 \mathrm{~cm}^{-1}, 1047.94 \mathrm{~cm}^{-1}, 947.74 \mathrm{~cm}^{-1}$, and $880.00 \mathrm{~cm}^{-1}$. The BSA NEDDS stored at room temperature for 180 days showed peaks at $3444.22 \mathrm{~cm}^{-1}$, $2975.21 \mathrm{~cm}^{-1}, 2539.36 \mathrm{~cm}^{-1}, 2412.35 \mathrm{~cm}^{-1}$, $2133.42 \mathrm{~cm}^{-1}, 1923.02 \mathrm{~cm}^{-1}, 1651.08 \mathrm{~cm}^{-1}$, $1519.29 \mathrm{~cm}^{-1}, 1455.12 \mathrm{~cm}^{-1}, 1416.10 \mathrm{~cm}^{-1}, 1383.80 \mathrm{~cm}^{-1}$, $1328.78 \mathrm{~cm}^{-1}, 1274.41 \mathrm{~cm}^{-1}, 1185.89 \mathrm{~cm}^{-1}, 1088.11 \mathrm{~cm}^{-1}$, $1048.26 \mathrm{~cm}^{-1}, 943.77 \mathrm{~cm}^{-1}$, and $880.10 \mathrm{~cm}^{-1}$. From these data, the BSA aqueous solution and the three samples stored at room temperature for 30,90 , and 180 days were mainly characterized by a band at around $1653 \mathrm{~cm}^{-1}$, which was assigned to the $\alpha$-helix conformation $\left(1658-1650 \mathrm{~cm}^{-1}\right)$ and can also be observed in free BSA $\left(1653.43 \mathrm{~cm}^{-1}\right)$. The peaks at $3435 \mathrm{~cm}^{-1}, 1640 \mathrm{~cm}^{-1}, 1415 \mathrm{~cm}^{-1}$, and $1109 \mathrm{~cm}^{-1}$ were due to the stretching of $\mathrm{O}-\mathrm{H}, \mathrm{COO}$ (asymmetric), $\mathrm{COO}$ (symmetric), and $\mathrm{C}-\mathrm{O}-\mathrm{C}$, respectively. The characteristic band of the BSA proteins at $1650 \mathrm{~cm}^{-1}$ and $1530 \mathrm{~cm}^{-1}$ are due to the $\mathrm{C}=\mathrm{O}$ stretching band of the carboxyl functional group on the 

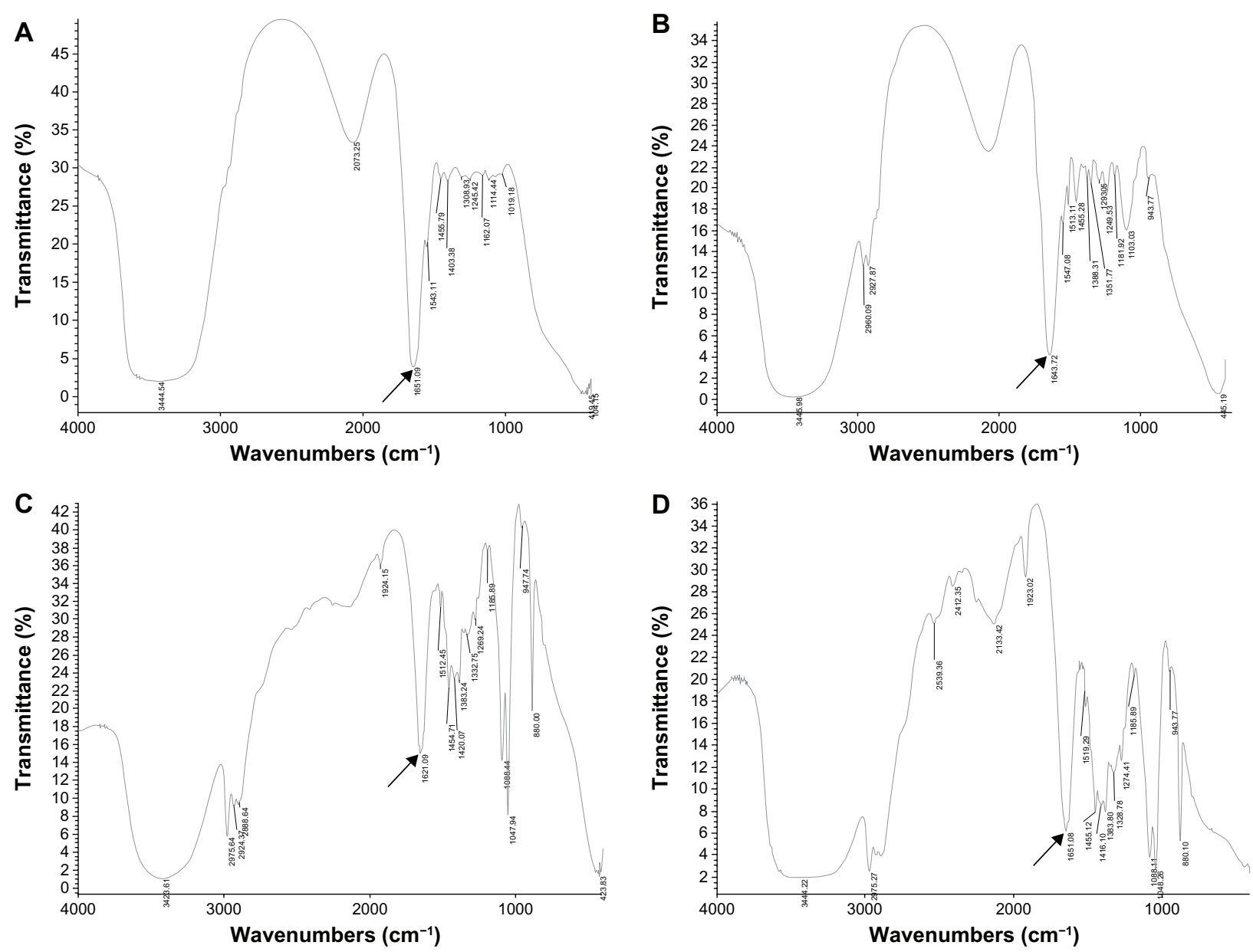

Figure 9 (A-D) Tertiary structural integrity results of the BSA in the NEDDS. Note: Arrows represent BSA absorption peaks.

Abbreviations: BSA, bovine serum albumin; NEDDS, nanoemulsion drug-delivery system.

tryptophan moiety as well as the carboxylate group. The FTIR spectroscopy results showed that the tertiary structure of BSA did not change in comparison with that of the BSA in the aqueous solution and the three samples stored at room temperature for 30,90 , and 180 days.

\section{Discussion}

In this study, we successfully developed the BSA NEDDS with a high encapsulation efficiency $(>90 \%)$ and drug-loading (45 $\mathrm{mg} / \mathrm{mL}$ ) using the phase-inversion method and the pseudoternary phase diagram. The entrapment efficiency and drug loading of this delivery system was measured by a microbicinchoninic acid assay, which is a traditional method for measuring the protein content of a drug. ${ }^{27}$ The BSA loaded into the nanoemulsion was separated by centrifugation, and the supernatant was analyzed by a microbicinchoninic acid assay. Centrifugation is normally used to determine the stability of a delivery system after emulsion/nanoemulsion formation. Importantly, it is a standard for distinguishing between nanoemulsion and traditional emulsions. It is also the easiest and most widely used and effective method for separating molecules. We converted the nanoemulsion to an emulsion with absolute ethanol and separated BSA by high-speed centrifugation $(13,000 \times g, 30$ minutes $)$. For comparison, soy protein isolate emulsions and gels were centrifuged in a microcentrifuge tube at $10,000 \times g$ for 20 minutes at room temperature. ${ }^{28}$ These results also confirmed that this delivery system is thermodynamically stable in these particular concentrations of oil, surfactant, and water, with no phase separation, creaming, or cracking. ${ }^{29}$

These results show that the stability of a protein drug can be improved greatly in our research, which we attribute to the following characteristics of this system. First, we used a novel nanoemulsion-drug delivery system, which is one of the most promising techniques for the delivery of drugs. Its advantages include higher storage stability and thermodynamic stability. ${ }^{30-32}$ We chose the nanoemulsion-drug delivery system formula to apply to a protein drug because 
Table I Fourier transform infrared spectra results of the bovine serum albumin (BSA) nanoemulsion drug-delivery system (NEDDS)

\begin{tabular}{|c|c|c|c|c|}
\hline Sample & $\begin{array}{l}\text { BSA } \\
\text { aqueous } \\
\text { solutions }\end{array}$ & $\begin{array}{l}\text { BSA } \\
\text { NEDDS } \\
\text { stored } \\
\text { for } 30 \text { days }\end{array}$ & $\begin{array}{l}\text { BSA } \\
\text { NEDDS } \\
\text { stored } \\
\text { for } 90 \text { days }\end{array}$ & $\begin{array}{l}\text { BSA } \\
\text { NEDDS } \\
\text { stored } \\
\text { for } 180 \text { days }\end{array}$ \\
\hline Peak I & 3445.54 & 3445.98 & 3423.61 & 3444.22 \\
\hline Peak 2 & 2073.25 & 2960.09 & 2975.64 & 2975.21 \\
\hline Peak 3 & 1651.09 & 2929.87 & 2924.37 & 2539.36 \\
\hline Peak 4 & I543.1I & 1643.72 & 2888.64 & 2412.35 \\
\hline Peak 5 & 1455.79 & 1547.08 & 1924.15 & 2133.42 \\
\hline Peak 6 & 1403.38 & 1513.11 & 1651.09 & 1923.02 \\
\hline Peak 7 & 1308.93 & 1455.28 & 1512.45 & 1651.08 \\
\hline Peak 8 & 1245.23 & $|388.3|$ & | 454.77 & 1519.29 \\
\hline Peak 9 & 1162.07 & $|35| .77$ & 1420.07 & 1455.12 \\
\hline Peak 10 & 1114.44 & 1293.05 & | 383.24 & 1416.10 \\
\hline Peak II & 1019.18 & 1249.53 & 1332.75 & 1383.80 \\
\hline Peak 12 & 409.45 & 1181.92 & 1269.24 & | 328.78 \\
\hline Peak 13 & $40 I .15$ & 1103.03 & 1185.99 & $|274.4|$ \\
\hline Peak 14 & & 943.77 & 1088.44 & 1185.89 \\
\hline Peak 15 & & 445.17 & 1047.94 & 1088.11 \\
\hline Peak 16 & & & 947.74 & 1048.26 \\
\hline Peak 16 & & & 880.00 & 943.77 \\
\hline Peak 17 & & & & 880.10 \\
\hline
\end{tabular}

Notes: Values are expressed as wavenumbers $\left(\mathrm{cm}^{-1}\right)$; peak values (bold, underscore) represent BSA absorbtion peaks.

it has a gel phase that forms through the preparation process. Second, we obtained the largest area of this nanoemulsion-drug delivery system by using pseudoternary diagrams to determine the best formula. Phase diagrams that provided information about the boundaries between different phases as a function of composition and other variables, such as temperature, were constructed. From this information, structural organization can be inferred. ${ }^{33}$ Therefore, the choice of oil and surfactant and the mixing ratio of oil to surfactant/cosurfactant are key issues in producing the nanoemulsion. The use of the phaseinversion method, a spontaneous low-emulsification-energy method also called the titration method, to prepare the BSA NEDDS is also important. Such systems can be prepared simply by mixing oil, water, surfactant, and cosurfactant in the appropriate proportions, followed by mild agitation. The order of component mixing is generally not critical, because the nanoemulsion-drug delivery system is formed spontaneously. Nevertheless, the driving forces are small, and it can take a long time for the system to reach equilibrium. ${ }^{34}$ Aqueous titration or spontaneous emulsification has been successfully investigated for preparing nanoemulsions of many lipophilic drugs in previous articles. ${ }^{34}$ Some studies on the nanoemulsion-delivery system have frequently used highenergy methods, especially homogenization. ${ }^{22}$ Homogenization pressure significantly influences the properties of emulsions because the shear forces and turbulence produced during homogenization, both of which are pressure-dependent, affect the particle size and size distribution. ${ }^{35}$ It has also been shown that disruption of any of these interactions will shift this delicate balance and destabilize the protein. The chemical and physical stability of proteins can also be compromised by environmental factors, such as $\mathrm{pH}$, ionic strength, temperature, high pressure, nonaqueous solvents, metal ions, detergents, adsorption, and agitation and shearing. ${ }^{36}$ We chose the phase-inversion method, which is a low-energy emulsification method, to avoid destabilizing the protein drug. Finally, the BSA NEDDS formula does not include any physical reagents such as inorganic solvents (such as methylene chloride or acetone) that might destroy the protein drug, in contrast to other delivery systems. We used simpler preparation techniques compared to those used to generate microparticles, nanospheres, and nanoparticles, which may also contribute to the enhanced stability of the protein drug.

It is known that the stability of protein drugs remains one of the key hurdles to their successful entry into the market. Therefore, many factors must be considered for these drugs: structural integrity and specificity of the primary, secondary, and tertiary protein structures, relative content, and biological activity. Many traditional methods are very sensitive and useful for evaluating the structural integrity and specificity of proteins, including centrifugation, micro-BCA method, sodium dodecyl sulfate polyacrylamide gel electrophoresis, Western blotting, enzyme-linked immunosorbent assay, circular dichroic spectrometry, and FTIR spectrometry. In this study, the blank nanoemulsion or emulsion did not interfere with the identification of BSA in the nanoemulsion. The essential characteristics and thermodynamic stability of the novel BSA NEDDS were also evaluated. BSA has been widely used with many drug-delivery systems such as nanoparticles, microparticles, emulsions, gels, and liposomes because it is a common and model protein. In this study, a novel BSA NEDDS was designed and successfully prepared by the phase-inversion method and using pseudoternary phase diagrams. The results of stability tests, including assessments of particle size and zeta potential under different temperatures or $\mathrm{pH}$ values and the integrity of the primary, secondary, and tertiary structures of BSA, have shown that the BSA NEDDS is very stable and greatly enhances the stability of the protein drug BSA. These data will enhance the reliability of the nanoemulsion-drug delivery system for use as an oral protein drug-delivery system and change the route of drug delivery, ie, injection, and the requirement of freezing drugs. In future, we intend to study some more advanced 
methods involving high-performance liquid chromatography, capillary electrophoresis, and protein drugs such as human serum albumin. Also, the stability of the BSA nanoemulsiondelivery system stored at different temperatures of $4^{\circ} \mathrm{C}$, $25^{\circ} \mathrm{C}, 40^{\circ} \mathrm{C}$, and $45^{\circ} \mathrm{C}$ and $65^{\circ} \mathrm{C}$ should be researched further.

\section{Conclusion}

In the present study, a NEDDS for oral administration of the standard-model protein drug BSA was successfully developed. The optimum nanoemulsion formulation was a mixture of Cremophor EL-35, propylene glycol, and isopropyl myristate at a ratio of 18:9:3 (w/w/w). The BSA NEDDS showed a narrow particle-size distribution, with a PDI value of 0.170 , a spherical average particle size of $21.8 \mathrm{~nm}$, and zeta potential of $-24.8 \mathrm{mV}$. This nanoemulsion-drug delivery system is very stable with high encapsulation efficiency and $45 \mathrm{mg} / \mathrm{mL}$ drug loading. It also ensured good integrity of the primary, secondary, and tertiary structures; the system showed good specificity and bioactivity when stored at room temperature for 180 days. These results show that the NEDDS can greatly enhance the stability of a protein drug while retaining good integrity, specificity, and bioactivity. Thus, this delivery system can greatly enhance stability and be employed for oral administration of protein drugs.

\section{Acknowledgments}

Thanks to China National Key Hi-Tech Innovation Project for the R\&D of Novel Drugs (No 2008ZX09101-033) and National Natural Science Foundation of China (No 81172892). We are grateful to the Shanghai Laboratory of Malvern Instruments, UK, for the zeta-potential and particle-size measurements. We are also grateful to Xueheng Yang of Chongqing University for detecting the BSA NEDDS by atomic-force microscopy.

\section{Disclosure}

The authors report no conflicts of interest in this work.

\section{References}

1. Capelle MA, Gurny R, Arvinte T. High throughput screening of protein formulation stability: practical considerations. Eur J Pharm Biopharm. 2007;65(2):131-148.

2. Sakulku U, Nuchuchua O, Uawongyart N, Puttipipatkhachorn S, Soottitantawat A, Ruktanonchai U. Characterization and mosquito repellent activity of citronella oil nanoemulsion. Int J Pharm. 2009; 372(1-2):105-111.

3. Almeida AJ, Souto E. Solid lipid nanoparticles as a drug delivery system for peptides and proteins. Adv Drug Deliv Rev. 2007;59(6):478-490
4. Lee KY, Yuk SH. Polymeric protein delivery systems. Prog Polym Sci. 2007;32(7):669-697.

5. Bilati U, Allémann E, Doelker E. Strategic approaches for overcoming peptide and protein instability within biodegradable nano- and microparticles. Eur J Pharm Biopharm. 2005;59(3):375-388.

6. Rathore N, Rajan RS. Current perspectives on stability of protein drug products during formulation fill and finish operations. Biotechnol $A d v$. 2008;24(3):504-514.

7. Carrillo-Nava E, Dohnal V, Costas M. Infinite dilution activity coefficients for toluene in aqueous solutions of the protein stabilizers glycerol, ethylene glycol, glucose, sucrose and trehalose. $J$ Chem Thermodyn. 2002;34(4):443-456.

8. Sun HW, Liu W, Zou QM. Helicobacter pylori vaccine delivery systems: research progress. J Int Pharm Res. 2009;36(6):431-434. Chinese.

9. Yang L, Cui F, Cun D, Tao A, Shi K, Lin W. Preparation, characterization and biodistribution of the lactone form of 10-hydroxycamptothecin (HCPT)-loaded bovine serum albumin (BSA) nanoparticles. Int $J$ Pharm. 2007;340:163-172.

10. Rodrigues MMA, Simioni AR, Primo FL, Siqueira-Mourab MP, Morais PC, Tedesco AC. Preparation, characterization and in vitro cytotoxicity of BSA-based nanospheres containing nanosized magnetic particles and/or photosensitizer. J Magn Magn Mater. 2009; 32(10):1600-1603.

11. Wang Y, Wang X, Luo G, Dai Y. Adsorption of bovin serum albumin (BSA) onto the magnetic chitosan nanoparticles prepared by a microemulsion system. Bioresour Technol. 2008;99(9):3881-3884.

12. Zhang C, Cheng Y, Qu G, et al. Preparation and characterization of galactosylated chitosan coated BSA microspheres containing 5-fluorouracil. Carbohydr Polym. 2008;72(3):390-397.

13. Kim BS, Oh JM, Kim KS, et al. BSA-FITC-loaded microcapsules for in vivo delivery. Biomaterials. 2009;32(10):902-909.

14. Sharma N, Bansal M, Visht S, Sharma PK, Kulkarni GT. Nanoemulsion: A new concept of delivery system. Chronicles of Young Scientists. 2010;1(2):2-6.

15. Chen $\mathrm{H}$, Khemtong $\mathrm{C}$, Yang X, Chang X, Gao J. Nanonization strategies for poorly water-soluble drugs. Drug Discov Today. 2011;16(7-8): 354-360.

16. Gao F, Zhang Z, Bu H, et al. Nanoemulsion improves the oral absorption of candesartan cilexetil in rats: performance and mechanism. $J$ Control Release. 2011;149(2):168-174.

17. Shafiq S, Shakeel F, Khar RK. Enhanced stability of ramipril in nanoemulsion containing cremophor-EL: a technical note. AAPS Pharm Sci Tech. 2008;9(4):1097-1101.

18. Shakeel F, Baboota S, Ahuja A, Ali J, Faisal MS, Shafiq S. Stability evaluation of celecoxib nanoemulsion containing Tween 80. Thai $J$ Pharm Sci. 2008;32:4-9.

19. Kong M, Park HJ. Stability investigation of hyaluronic acid based nanoemulsion and its potential as transdermal carrier. Carbohydr Polym. 2011;83(3):1303-1310.

20. Kong M, Chen X, Park HJ. Design and investigation of nanoemulsified carrier based on amphiphile-modified hyaluronic acid. Carbohydr Polym. 2011;83(2):462-469.

21. Wang L, Li X, Zhang G, Dong J, Eastoe J. Oil-in-water nanoemulsions for pesticide formulations. J Colloid Interface Sci. 2007;314(1): 230-235.

22. Yuan Y, Gao Y, Zhao J, Mao L. Characterization and stability evaluation of $\beta$-carotene nanoemulsions prepared by high pressure homogenization under various emulsifying conditions. Food Res Int. 2008;41(1):61-68.

23. Silva HD, Cerqueira MA, Souza BWS, et al. Nanoemulsions of $\beta$-carotene using a high-energy emulsification-evaporation technique. J Food Eng. 2011;102:130-135.

24. Klang V, Matsko N, Zimmermann AM, Vojnikovic E, Valenta C. Enhancement of stability and skin permeation by sucrose stearate and cyclodextrins in progesterone nanoemulsion. Int J Pharm. 2010; 393(1-2):152-160. 
25. Baker JR Jr, Bielinska A, Landers J, Janczak K, Cao P. Enhanced systemic and mucosal immune responses in mice immunized with recombinant Bacillus anthracis protective antigen (rPA) using a novel nanoemulsion adjuvant. J Allergy Clin Immunol. 2004;113(2):S292.

26. Shafiq S, Sushma T, Ahmad FJ. Design and development of oral in water rampipril nanoemulsion formulation: in vitro and in vivo evaluation. J Biomed Nanotechnol. 2007;3:28-44.

27. Gutierro I, Hernández RM, Igartua M, Gascón AR, Pedraz JL. Size dependent immune response after subcutaneous, oral and intranasal administration of BSA loaded nanospheres. Vaccine. 2002;21(1-2): 67-77.

28. Xin Gu, Lydia J, Euston SR. Effects of different oils on the properties of soy protein isolate emulsions and gels. Food Res Int. 2009;42(8): 925-932.

29. Shafiq S, Shakeel F. Effect of Labrasol on self-nanoemulsification efficiency of ramipril nanoemulsion. Pharmazie. 2009;64(12): 812-817.

30. Shakeel F, Ramadan W, Ahmed MA. Investigation of true nanoemulsions for transdermal potential of indomethacin: characterization, rheological characteristics, and ex vivo skin permeation studies. J Drug Target. 2009; 17(6):435-441
31. Bali V, Ali M, Ali J. Novel nanoemulsion for minimizing variations in bioavailability of ezetimibe. J Drug Target. 2001;18(7):506-519.

32. Patel AR, Vavia PR. Preparation and in vivo evaluation of SMEDDS (self-microemulsifying drug delivery system) containing fenofibrate. AAPS Pharm Sci Tech. 2007;9(3):E344-E352.

33. Shafiq-un-Nabi S, Shakeel F, Talegaonkar S, et al. Formulation development and optimization using nanoemulsion technique: a technical note. AAPS Pharm Sci Tech. 2007;8(2):E12-E17.

34. Shafiq-un-Nabi S, Shakeel F, Talegaonkar S, et al. Formulation development and optimization using nanoemulsion technique: a technical note. AAPS Pharm Sci Tech. 2007;2(8):28-32.

35. Floury J, Desrumaux A, Lardières J. Effect of high-pressure homogenization on droplet size distributions and rheological properties of model oil-in-water emulsions. Innov Food Sci Emerg Technol. 2000 1(2):127-134

36. Wang W. Instability, stabilization, and formulation of liquid protein pharmaceuticals. Int J Pharm. 1999;185(2):129-188.
International Journal of Nanomedicine

\section{Publish your work in this journal}

The International Journal of Nanomedicine is an international, peerreviewed journal focusing on the application of nanotechnology in diagnostics, therapeutics, and drug delivery systems throughout the biomedical field. This journal is indexed on PubMed Central,

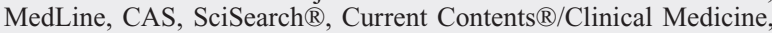

\section{Dovepress}

Journal Citation Reports/Science Edition, EMBase, Scopus and the Elsevier Bibliographic databases. The manuscript management system is completely online and includes a very quick and fair peer-review system, which is all easy to use. Visit http://www.dovepress.com/ testimonials.php to read real quotes from published authors. 